\title{
The dust budget crisis in high-redshift submillimetre galaxies
}

\author{
Kate Rowlands ${ }^{* \dagger}$ \\ (SUPA) School of Physics \& Astronomy, University of St Andrews, St Andrews, KY16 9SS, UK \\ E-mail: ker7@st-andrews.ac.uk
}

\section{Haley Gomez}

School of Physics \& Astronomy, Cardiff University, The Parade, Cardiff, CF24 3AA, UK

E-mail: haley.gomezlastro.cf.ac.uk

\section{Loretta Dunne, Steve Maddox}

Department of Physics and Astronomy, University of Canterbury, Christchurch, New Zealand

E-mail: loretta.dunnedcanterbury.ac.nz,

steve.maddoxdcanterbury.ac.nz

\section{Alfonso Aragón-Salamanca, Simon Dye}

School of Physics \& Astronomy, The University of Nottingham, Nottingham, NG7 2RD, UK

E-mail: Alfonso.Aragon@nottingham.ac.uk, Simon. Dye@nottingham.ac.uk

\section{Elisabete da Cunha}

Max Planck Institute for Astronomy, Konigstuhl 17, 69117, Heidelberg, Germany

E-mail: cunha@mpia-hd.mpg.de

\section{Paul van der Werf}

Leiden University, P.O. Box 9500, 2300 RA Leiden, The Netherlands

E-mail: pvdwerfestrw.leidenuniv.nl

We apply a chemical evolution model to investigate the sources and evolution of dust in a sample of 26 high-redshift $(z>1)$ submillimetre galaxies (SMGs) with complete photometry from the UV-submillimetre. Models with dust produced only by low-intermediate mass stars fall a factor 240 short of the observed dust masses of SMGs, the well-known 'dust-budget crisis'. Adding an extra source of dust from supernovae can account for the dust mass in $19 \%$ of the sample. After accounting for dust produced by supernovae the remaining deficit in the dust mass provides support for higher supernova yields or substantial grain growth in the interstellar medium. Efficient destruction of dust by supernova shocks increases the tension between the model and observed dust masses. Models which best reproduce the physical properties of SMGs have a rapid build-up of dust from both stellar and interstellar sources and minimal dust destruction.

The Life Cycle of Dust in the Universe: Observations, Theory, and Laboratory Experiments - LCDU 2013, 18-22 November 2013

Taipei, Taiwan

\footnotetext{
*Speaker.

${ }^{\dagger}$ Supported by the European Research Council Starting Grant (P.I. V. Wild).
} 


\section{Introduction}

The first blind submillimetre surveys discovered a population of highly star-forming (100 $1000 \mathrm{M}_{\odot} \mathrm{yr}^{-1}$ ), dusty galaxies at high redshift [1]. These submillimetre galaxies (SMGs) are thought to be undergoing intense, obscured starbursts [2], which may be driven by streams of cold gas [3] or gas-rich major mergers [4]. The source of interstellar dust in SMGs is still a controversial issue, particularly whether it originates from the cool, stellar winds of low-intermediate mass stars (LIMS), or supernovae ( $\mathrm{SNe}$ ). Many studies have revealed a 'dust budget crisis' [5 - 10], whereby it is difficult to explain the high dust masses observed in high redshift galaxies through dust from LIMS. The surprisingly constant dust-to-metals ratio measured in galaxies over a wide range of cosmic time also indicates that a rapid mechanism of dust formation is needed [11], requiring dust formation timescales to be the same order as the metal enrichment timescale. Due to their short lifetimes, massive-star SNe have long been proposed as a potential source of dust at early times $[5,12,6,13]$. There is now little doubt that dust is formed in SN ejecta [14 - 16] though the amount of dust which will ultimately survive the supernova shocks is highly uncertain [17]. Additionally, dust grain growth in the ISM has been proposed as an extra source of dust in galaxies $[7,8,18,19]$, which could make up the shortfall in the dust budget of galaxies. The difficulty with determining the origin of dust in galaxies and its lifecycle arises due to a lack of large samples of sources with which to test these issues.

We selected a sample of SMGs at $1.0<z<5.3$ with far-infrared photometry spanning the peak of the dust emission from [20] (see also [21]). Galaxy properties were derived for the population by fitting their spectral energy distributions (SEDs) from the UV to the submillimetre in a consistent way using the MAGPHYS code [22]. The SMGs have an average star-formation rate (SFR) of $390_{-70}^{+80} \mathrm{M}_{\odot} \mathrm{yr}^{-1}$ and a dust mass of $1.2_{-0.2}^{+0.3} \times 10^{9} \mathrm{M}_{\odot}$. Here, we investigate the origin of dust in these high redshift SMGs using an updated version of the chemical evolution model of Morgan \& Edmunds [5] which incorporates realistic star-formation histories (SFHs) for each galaxy, with greater complexity than previous chemical evolution studies have attempted. The sample properties and derivation of the observational parameters are described in detail in [21] (see also [20]).

\section{The Chemical Evolution Model}

In order to investigate the origin of dust in our sample of high redshift galaxies, we compare the observed dust masses of SMGs to predictions using an updated version of the chemical evolution model of [5]. The model is fully described in [23] and is based on chemical evolution models in the literature $[24,25]$. By relaxing the instantaneous recycling approximation to account for the lifetimes of stars of different masses, the model tracks the build-up of heavy elements over time produced by stars (LIMS and SNe) where some fraction of the heavy elements will condense into dust. Given an input SFH, gas is converted into stars over time, assuming a Chabrier (2003) [26] initial mass function. To model the SMGs we consider a closed box model, assuming no inflow or outflow of gas or metals. The initial gas mass is set at $2 \times$ the best-fit stellar mass derived from the SED fitting, such that at the end of the SFH history, $\sim 50 \%$ of the total galaxy mass ends up in stars, in agreement with observations [27]. By design, the final stellar masses are in close agreement with the best-fit stellar masses (mean $M_{*}=6.3_{-1.3}^{+1.6} \times 10^{10} \mathrm{M}_{\odot}$ ) derived from the SED fitting [21]. 
Previous studies of chemical evolution in SMGs [5] often assumed a SFR proportional to the gas mass which decreased smoothly with time. One of the main differences between this work and [5] (among others) is the incorporation of a more realistic SFH with bursts of variable strength and duration, and with an underlying SFH which can be either exponentially rising or declining. The adopted SFH gives a physically plausible representation of the SFH which is consistent with the physical properties of each SMG, which we can use as an input to our chemical evolution models.

\section{The origin of dust in SMGs}

We now explore how well different chemical evolution models can reproduce the dust masses of the SMGs, and other observational properties of SMGs as a population. We first consider dust production in a closed box from LIMS only for each SMG in the sample, assuming no dust destruction as an optimistic case. In Fig. 1 (a) we show the difference between the final dust mass derived from the chemical evolution modelling and the observed dust mass from the SED fitting. The dust masses from the chemical evolution model with dust from LIMS fall far short of the observed dust masses for the majority of SMGs. On average, the theoretical dust masses are $5.0 \times 10^{6} \mathrm{M}_{\odot}$, which is a factor 240 lower than the average observed dust mass in the SMG sample $\left(1.2_{-0.2}^{+0.3} \times 10^{9} \mathrm{M}_{\odot}\right)$. This provides definitive evidence that (without changing the IMF) the majority of dust in SMGs must come from a source other than LIMS. Although noted by previous authors, this was based previously on smaller samples and/or simple parameterized SFHs [13, 8]. For the closed box model, the median metallicity for the SMGs reaches $0.9 \mathrm{Z}_{\odot}$ in agreement with observations. The median fraction of metals in the ISM in the form of dust $\left(\eta_{Z}=M_{d} / M_{Z}\right)$ in the LIMS-only model is $0.4 \%$; this is well below the dust-to-metals ratio of $\sim 50 \%$ observed out to $z \sim 6$ [11]. This indicates that additional dust input is needed from stellar or interstellar sources.

If we include dust production from both SNe and LIMS, dust builds up more rapidly in SMGs with a delay of only tens of Myrs between the highest mass stars forming and evolving to the SN phase. Adding dust from $\mathrm{SNe}$ accounts for more than an order of magnitude increase in the dust mass of SMGs (with a median mass of $1.9 \times 10^{8} \mathrm{M}_{\odot}$ for the LIMS+SNe model) compared to the dust mass from LIMS only $\left(5.0 \times 10^{6} \mathrm{M}_{\odot}\right)$. The model dust masses using LIMS and SNe match the observed values (accounting for the \pm 0.2 dex uncertainty in the MAGPHYS-derived dust masses) in $\sim 19 \%$ of cases. The median metallicity of the SMGs in this model is the same as with LIMS only $\left(0.9 \mathrm{Z}_{\odot}\right)$, but with the inclusion of $\mathrm{SN}$ dust the dust-to-metals ratio is higher $\left(\eta_{Z}=16 \%\right)$. In Fig. 1 (b) it can be seen that the predicted dust masses for the majority of the SMGs falls short of the observed dust masses, which indicates additional sources of dust, or even higher SN dust yields than used in this work are needed.

In Fig 1 (c) we consider the extreme case of maximal dust production from $\mathrm{SNe}$ such that all metals ejected in this phase are incorporated into dust. Sufficiently high dust masses are achieved in this scenario (a median of $7.6 \times 10^{8} \mathrm{M}_{\odot}$ ) to account for the observed dust in 15/26 SMGs. The gas-to-dust ratio for the maximal SN dust model is in agreement with observed values for SMGs of $30-50$ [28]. The resulting median dust-to-metals ratio $\eta_{Z} \sim 68 \%$, somewhat higher than the observed values of $\sim 0.5$ [11]. Far-infrared observations of supernova remnants (SNRs) indicate high condensation efficiencies, suggesting that at some point the majority of heavy elements produced in $\mathrm{SN}$ ejecta can condense into dust. However, evidence for efficient dust production is observed 

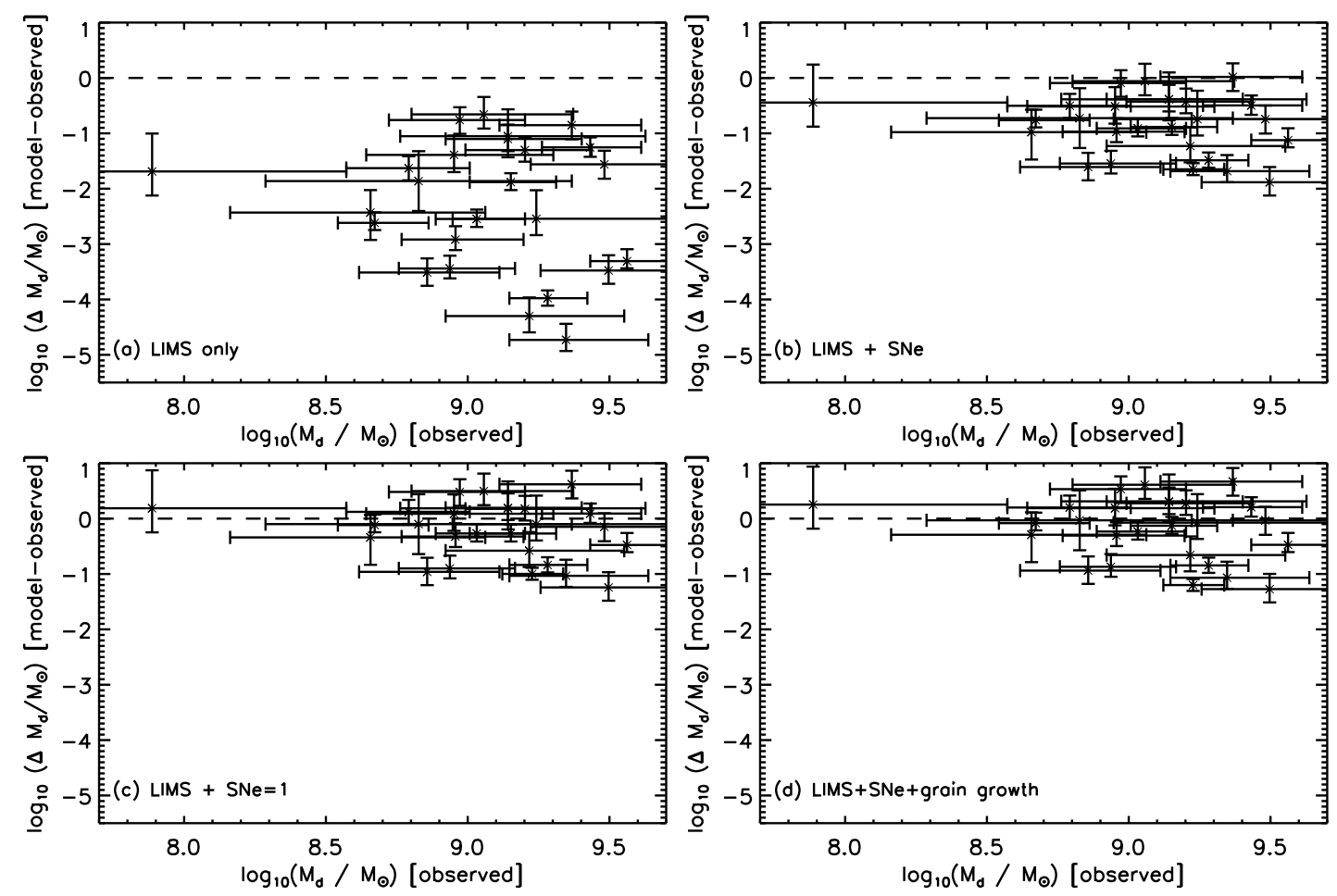

Figure 1: The difference between the dust masses derived from the chemical evolution models and the observed dust masses for the high redshift SMGs, assuming no dust destruction. Dust is produced in panel (a) by low-intermediate mass stars (LIMS) only, LIMS and supernovae (b), LIMS and maximal supernova dust contribution (all metals into dust) (c), and by LIMS, SNe and grain growth (d).

in only a handful of SNRs, and it is unclear how much dust will survive passage into the ISM. It is therefore still possible that $\mathrm{SN}$ dust is unable to account for all the dust in galaxies, i.e. either a significant mass of dust must form rapidly in the ISM or theoretical metal yields from SNe obtained from stellar evolution models are systematically underestimated.

There are large uncertainties about the effectiveness of dust destruction in the ISM, yet it is often used in chemical evolution analyses. If SN shocks are efficient in destroying dust, then assuming $1000 \mathrm{M}_{\odot}$ of ISM $\left(m_{\mathrm{ISM}}\right)$ is cleared by each supernova event, the dust mass we obtain is reduced by a factor of 6-10 on average if dust is produced by LIMS and LIMS+SNe, respectively. This compounds the dust budget crisis further. If dust is produced by both LIMS and SNe, then any increase in the dust mass by including $\mathrm{SNe}$ is effectively cancelled out by the dust destroyed, resulting in a median dust mass of $1.8 \times 10^{7} \mathrm{M}_{\odot}$, approximately 3.6 times the mass obtained with the LIMS only model $\left(5 \times 10^{6} \mathrm{M}_{\odot}\right)$. If dust is shielded in cold, dense regions of the ISM, then it is possible that the dust destruction efficiency of SN shocks will be reduced $[6,9]$ with $m_{\text {ISM }}=$ $100 \mathrm{M}_{\odot}$ more appropriate given the increased density of the ISM gas in SMGs. Including dust destruction in the models with $m_{\mathrm{ISM}}=100 \mathrm{M}_{\odot}$ lowers the dust mass by a factor of 1.2-1.6 (for the LIMS only and LIMS+SNe models). A cautionary note here is that theoretical dust destruction models appear to be too efficient at destroying dust grains. The fact that we observe so much dust in galaxies, including those with very little recent star formation [29], implies the dust destruction rate must be balanced by the injection rate from stars and grain growth in the ISM [19]. 
Adding grain growth to the LIMS only model (with no dust destruction) fails to adequately reproduce the observed dust masses for the majority of SMGs. In Fig 1 (d) we therefore consider dust produced by LIMS, SNe and grain growth in the ISM. Including grain growth on average increases the dust mass by a factor of 200 to $\simeq 9.8 \times 10^{8} \mathrm{M}_{\odot}$, compared to a model in which dust is contributed by LIMS only. Grain growth can therefore make up the shortfall in the predicted dust masses for $62 \%$ of SMGs in the sample, but only if grain growth is the dominant form of dust production in SMGs and there is no significant dust destruction. However, in this scenario, a large fraction $(77 \%)$ of the metals is locked up in the form of dust which is higher than typically observed. This problem could indicate that metal yields of stars are underestimated.

\section{Conclusions}

We used a chemical evolution model to reproduce the properties of 26 high-redshift $(z>1)$ SMGs. Our chemical model utilises a detailed treatment of the dust sources and sinks in galaxies and complex SFHs consistent with the UV-submillimetre SEDs. Models where dust is produced by LIMS only, and those which have efficient dust destruction result in dust-to-stellar mass ratios and/or gas-to-dust ratios which are inconsistent with observations of SMGs. Models which best match the physical properties of SMGs include rapid dust build-up from grain growth and supernova dust sources. In summary, the models which best reproduce the observed properties of SMGs are LIMS+SNe+grain growth and LIMS+maximal SN dust production (unphysical since this requires all of the SN metals to be in the form of dust). A modest amount of dust destruction can be accommodated if dust is produced by both stellar and interstellar sources.

\section{References}

[1] Barger A. J., et al., Submillimetre-wavelength detection of dusty star-forming galaxies at high redshift, Nature 3846690.

[2] Tacconi, L. J. et al., High-Resolution Millimeter Imaging of Submillimeter Galaxies, ApJ 640228 [arXiv:astro-ph/0511319].

[3] Davé, R., et al, The nature of submillimetre galaxies in cosmological hydrodynamic simulations, MNRAS 4041355 [arXiv:0909.4078].

[4] Engel, H. et al., Most Submillimeter Galaxies are Major Mergers, ApJ 724233.

[5] Morgan H. L., Edmunds M. G., Dust formation in early galaxies, MNRAS 343427 [arxiv:astro-ph/0302566].

[6] Dwek, E. and Galliano, F. and Jones, A. P., The Evolution of Dust in the Early Universe with Applications to the Galaxy SDSS J1148+5251, ApJ 662 927 [arXiv: 070 5.3799].

[7] Michałowski, M. J., Watson, D. and Hjorth, J., Rapid Dust Production in Submillimeter Galaxies at $z>4$ ?, ApJ 712942 [arXiv:1002.2636].

[8] Dunne, L. et al, Herschel-ATLAS: rapid evolution of dust in galaxies over the last 5 billion years, MNRAS 4171510 [arXiv: 1012.5186 ].

[9] Gall, C., Andersen, A. C. and Hjorth, J., Genesis and evolution of dust in galaxies in the early Universe. I. Modelling dust evolution in starburst galaxies, A\&A 528 A13 [arXiv:1011.3157]. 
[10] Valiante, R., Schneider, R., Salvadori, S. and Bianchi, S., The origin of the dust in high-redshift quasars: the case of SDSS J1148+5251, MNRAS 4161916 [arXiv: 1106.1418 ].

[11] Zafar T., Watson D., The metals-to-dust ratio to very low metallicities using GRB and QSO absorbers; extremely rapid dust formation, A\&A [arxiv:1303.1141].

[12] Dunne, L., Eales, S., Ivison, R., Morgan, H. and Edmunds, M., Type II supernovae as a significant source of interstellar dust, Nature $\mathbf{4 2 4} 285$ [arXiv: astro-ph/ 0307320 ].

[13] Gall, C., Hjorth, J. and Andersen, A. C., Production of dust by massive stars at high redshift, A\&A Rev. 1943 [arXiv: 1108.0403 ].

[14] Barlow M. J., et al., 2010, A Herschel PACS and SPIRE study of the dust content of the Cassiopeia A supernova remnant, $A \& A \mathbf{5 1 8} \mathrm{L} 138$ [arXiv: 1005.2688$].$

[15] Matsuura, M. et al., Herschel Detects a Massive Dust Reservoir in Supernova 1987A, Science 333 1258 [arXiv:1107.1477].

[16] Gomez, H. L. et al., A Cool Dust Factory in the Crab Nebula: A Herschel Study of the Filaments, ApJ 76096 [arXiv: 1209.5677$]$.

[17] Jones, A. P. and Nuth, J. A., Dust destruction in the ISM: a re-evaluation of dust lifetimes, A\&A 530 A44.

[18] Hirashita, H. and Kuo, T.-M., Effects of grain size distribution on the interstellar dust mass growth, MNRAS 4161340 [arXiv: 1105.4930$].$

[19] Mattsson, L. and Andersen, A. C., On the dust abundance gradients in late-type galaxies - II. Analytical models as evidence for massive interstellar dust growth in SINGs galaxies, MNRAS 42338 [arXiv: 1201.3374$]$.

[20] Magnelli B., et al., A Herschel view of the far-infrared properties of submillimetre galaxies, A\&A 539 155 [arxiv:1303.1141].

[21] Rowlands K., et al., H-ATLAS: Properties of dusty massive galaxies at low and high redshifts, MNRAS submitted.

[22] da Cunha E., Charlot S., Elbaz D., A simple model to interpret the ultraviolet, optical and infrared emission from galaxies, MNRAS 3881595 [arxiv: 0806.1020].

[23] Rowlands K., Gomez, H. L., Dunne, L., Aragón-Salamanca, A., Dye, S. Maddox, S., da Cunha, E., van der Werf, P., The dust budget crisis in high-redshift submillimetre galaxies, MNRAS submitted.

[24] Tinsley, B. M., Evolution of the Stars and Gas in Galaxies, FCP 5287.

[25] Dwek, E., The Evolution of the Elemental Abundances in the Gas and Dust Phases of the Galaxy, ApJ 501643 [arXiv:astro-ph/9707024].

[26] Chabrier G., Galactic Stellar and Substellar Initial Mass Function, PASP 115763 [arXiv:astro-ph/0304382].

[27] Bothwell, M. S. et al., A survey of molecular gas in luminous sub-millimetre galaxies, MNRAS 429 3047 [arXiv:1205.1511].

[28] Swinbank, M. et al., An ALMA Survey of Submillimetre Galaxies in the Extended Chandra Deep Field South: The Far-Infrared Properties of SMGs, MNRAS [arXiv:1310.6362].

[29] Rowlands K. et al., Herschel-ATLAS/GAMA: dusty early-type galaxies and passive spirals, MNRAS 4192545 [arXiv: 1109.6274$].$ 